\title{
Celestial pole offsets from lunar laser ranging and comparison with VLBI
}

\author{
W. Zerhouni and N. Capitaine \\ SYRTE, Observatoire de Paris, CNRS, UPMC, 61 avenue de l'Observatoire, 75014 Paris, France \\ e-mail: [wassila.zerhouni; nicole.capitaine]@obspm.fr
}

Received 5 June 2009 / Accepted 2 September 2009

\begin{abstract}
Context. The analysis of lunar laser ranging (LLR) observations is based on determining the round- trip travel times of light pulses between stations on the Earth and reflectors on the surface of the Moon. Several works have demonstrated that this technique is powerful in various domains including astronomy, geodynamics and gravitational physics.

Aims. In the field of geodynamics, LLR contributes to the realization of a dynamical celestial reference frame, in contrast to very long baseline interferometry (VLBI) that determines a kinematical celestial reference frame. In this paper, we have determined corrections to the celestial pole coordinates, denoted $X, Y$, using LLR observations. This determination is of particular interest for comparison with the one obtained from VLBI observations. The main purpose is to study the benefits of LLR for the determination of the celestial pole coordinates and second how to best combine the time series obtained from both techniques.

Methods. For these determinations, data acquired by LLR tracking stations since 1969 were analyzed and corrections to the nutation terms estimated using a weighted least square fit. Finally, LLR data were combined with the IVS combined VLBI series of 23-year duration.

Results. We have demonstrated the possibility of determining the celestial pole offsets from LLR data even though the results are not as accurate nor as dense as those obtained with VLBI. This work provides some external constraints to the celestial pole coordinates derived from VLBI observations. Moreover, the LLR determination of the long periodic nutation terms shows an improvement with respect to previous studies. The combination of LLR and VLBI series may indicate that the combined series reveal details that do not appear in the VLBI series alone.
\end{abstract}

Key words. astrometry - reference systems - Moon - ephemerides

\section{Introduction}

Various space geodetic techniques contribute to a better understanding and modeling of the Earth's rotation. Very long baseline interferometry (VLBI) plays an unquestionable role in determining the Earth orientation parameters (EOP). This technique is powerful especially for the determination of UT1 and the celestial pole coordinates. However, it has its limitations, and with the current level of accuracy, errors at a microarcsecond level from VLBI have to be considered. These errors can occur from the models used in the data reduction, as well as from the method of analyzing the data and from the realization of the reference systems. VLBI orients the terrestrial coordinate system with respect to the celestial coordinate system, the latter being realized by radio source positions. Thus, the realized reference frame depends on the choice of these radio sources as well as on the constraints applied. The satellite space-geodetic techniques, such as the global positioning system (GPS) or satellite laser tracking (SLR), also define an inertial reference frame. However, the imperfection of the models of the non-gravitational forces acting on the satellites gives inaccuracies in the determination of the precession of the ascending node on the long periods and thus in the realized reference frame. In contrast, lunar laser ranging (LLR) is the only space-geodetic technique capable to realize a dynamical reference system defined by the lunar orbit, which is determined with sufficient accuracy to allow a stable reference frame.
LLR has been operating since 1969 and has brought remarkable results in different fields such as lunar science, solar system dynamics, geophysics and fundamental physics. In the field of geodynamics, the lunar motion defines intrinsically a dynamical celestial reference system and contributes to the realization of both terrestrial and selenocentric reference frames. Several works have already been carried out in this field, especially concerning the estimation of the EOP. Williams et al. (1991) estimated the luni-solar precession from lunar laser ranges, Folkner et al. (1994) and Charlot et al. (1995) determined the precession and nutation components using a joint analysis of LLR and VLBI observations, and Chapront et al. (2002) estimated the general precession in longitude. This work contributes to this topic; it is of particular interest to see the potential of the LLR observations for the determination of the celestial pole coordinates denoted $(X, Y)$ in the Geocentric Celestial Reference System (GCRS), independent of any other technique.

LLR data and their analysis are described in Sects. 2 and 3, respectively. The strategy adopted for the calculation of the corrections to the celestial pole coordinates $D X, D Y$ with respect to the IAU 2006-2000A model of precession nutation using LLR observations is presented in Sect. 4 and the $D X, D Y$ time series obtained are analyzed. In Sect. 5 an analysis of the celestial pole offsets obtained from VLBI observations is presented for comparison with LLR results. The results of this comparison are discussed in Sect. 6.

As mentioned earlier, LLR contributes to the realization of a dynamical celestial reference frame, in contrast to VLBI that 


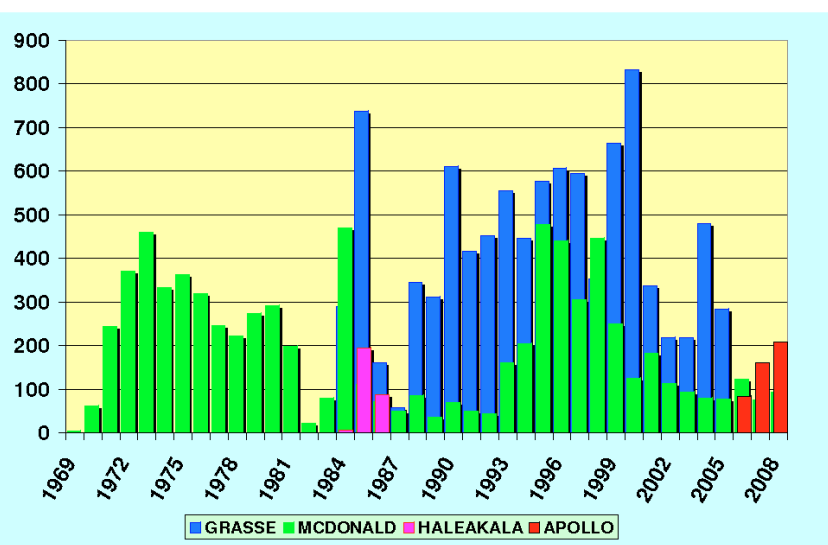

Fig. 1. Evolution of the number of LLR observations for each station from 1969 to 2008.

realizes a kinematical celestial reference frame. Even if the resolution of LLR data is not as good as VLBI, combining the series of celestial pole offsets obtained from both techniques can be stronger than either individually. Hence, a joint analysis of LLR and VLBI data by combining both series is presented in Sect. 7 .

\section{Lunar laser ranging data}

The principle of the lunar laser ranging technique is based on time propagation measurements of a light pulse between stations on the surface of the Earth and reflectors on the Moon.

An observation consists of a time delay; it is represented by a "normal point", which is built on the number of the photons detected on the way back to Earth, averaged over a session of observation of about ten minutes. The number of photons detected (called echos) vary from station to station. It mainly depends on the atmospheric conditions and on the period of lunation.

During the first $15 \mathrm{yr}$ of observation (i.e. 1969-1984), only McDonald station provided LLR measurements. The accuracy was of 25 to $30 \mathrm{~cm}$ in 1975 , but was improved to 10 to $15 \mathrm{~cm}$ in the 1980 s. Since 1985, it has achieved the current accuracy that is of the centimeter level.

The Grasse station (in France), currently a part of the "Observatoire de la Côte d'Azur", started LLR observations in 1982 that were interrupted between 2006 and 2009 for renovation. Another station in Hawaï (Haleakala) carried out lunar laser ranging from 1984 until 1990. Besides the McDonald and Grasse stations, the new Appolo station in southern New Mexico started LLR observations in 2006 and is still in operation (see Fig. 1).

LLR data depend on many parameters, such as the precision of the start detector, the precision of the return detector, the atmosphere, etc. One of the main factors that affects the precision of the measurement is the orientation of the reflector array that depends on the lunar libration. It can produce an uncertainty in the time delay of about 350 picoseconds for the largest reflector (Apollo XV) (cf. Samain et al. 1998).

\section{Lunar laser ranging data analysis: calculation of the residuals}

Like all the astro-geodetic techniques, the principle of the analysis is based on comparing the models with the observations, i.e. for LLR, comparing the observed value of the time delay
$\left(\Delta t_{\mathrm{o}}\right)$ with its computed value $\left(\Delta t_{\mathrm{c}}\right): \rho=\mathrm{O}-\mathrm{C}=\Delta t_{0}-\Delta t_{\mathrm{c}}$, with:

$\Delta t_{\mathrm{c}}=2 / c \times D$

$c$ being the speed of light and $D$ the distance station-reflector.

The propagation time has to be corrected taking into account several effects, such as the relativistic curvature of the light beam, the Earth's tidal deformations and the influence of the troposphere.

Furthermore, the computation of the distance $D$ involves the knowledge of the coordinates of the vectors $\boldsymbol{S E}, \boldsymbol{E} \boldsymbol{M}$ and $\boldsymbol{M R}$ in the Barycentric Celestial Reference System (BCRS), which are such that:

$D=S E+E M+M R$,

where $\mathrm{S}$ is the station, $\mathrm{E}$ the center of mass of the Earth, $\mathrm{M}$ the center of mass of the Moon and R the reflector.

The coordinates of the vector EM are provided by a lunar ephemeris; the coordinates of the Earth-Moon barycenter in the BCRS are provided by a planetary ephemeris. In our work, we have used, for the orbital motion of the Moon, the lunar ephemeris ELP2000 developed by Chapront-Touzé \& Chapront (1983), which is based on an analytical solution and numerical complements. For the motion of the Earth-Moon barycenter, JPL (Jet Propulsion Laboratory) planetary ephemerides (DE245) have been used. We chose DE245 in order to be consistent with the numerical complements used in ELP2000. We have also checked that the use of a more recent ephemeris gives LLR residuals of the same order.

The coordinates of the vector $\boldsymbol{M R}$ implies knowledge of the selenocentric coordinates of the reflectors and an ephemeris of the lunar librations. Those are given, in our case, by the analytical model for the libration of the Moon (cf. Moons 1982). Relativistic transformations of time scales have to be considered in order to express the various components in the same time scale. Relativistic corrections also have to be applied for the coordinate transformation between terrestrial and selenocentric reference systems to the BCRS. All the corrections described above have been applied according to Chapront et al. (1999).

The coordinates of the vector $\boldsymbol{S E}$ in the terrestrial reference system used in this work are those given in the ITRF2000. In order to determine these coordinates in a celestial reference system, we need a precise model for the Earth's rotation (see IERS Conventions 2003 for more details) ${ }^{1}$. In this work, we have used the Celestial Intermediate Origin (CIO) based transformation described in the IERS Conventions 2003, using the IAU20062000A precession nutation model as implemented in the SOFA $(2007)^{2}$ routines. This includes the use of the $x, y$ coordinates of the Celestial Intermediate Pole (CIP) in the International Terrestrial Reference System (ITRS) and UT1, which are given by the IERS C04 series.

To calculate the residuals, we have used 16355 normal points obtained by the LLR stations of (i) McDonald from 1969 to 2006; (ii) Haleakala from 1984 to 1990; and (iii) Grasse from 1984 to 2005. Table 1 shows the evolution of the LLR residuals obtained for the distance between the stations on the Earth and the reflectors on the Moon. It provides the root mean square (rms) of the residuals obtained with the data of each station, and the number N of LLR "normal points". Figures 2 and 3 show the residuals obtained for the Grasse and McDonald stations, respectively.

\footnotetext{
1 IERS technical Note 32: IERS Conventions (2003).

2 SOFA: http://www.iau-sofa.rl.ac.uk
} 
Table 1. Evolution of the quality of the LLR residuals for each station.

\begin{tabular}{ccrr}
\hline \hline Observatory & Period of observation & $\begin{array}{r}\text { rms } \\
(\mathrm{cm})\end{array}$ & $N$ \\
\hline McDonald & $1969-1986$ & 46 & 3522 \\
& $1987-2006$ & 9.2 & 3080 \\
Haleakala & $1984-1990$ & 11.1 & 481 \\
Grasse & $1984-1986$ & 24.6 & 1130 \\
& $1987-2005$ & 5.1 & 8142 \\
\hline
\end{tabular}

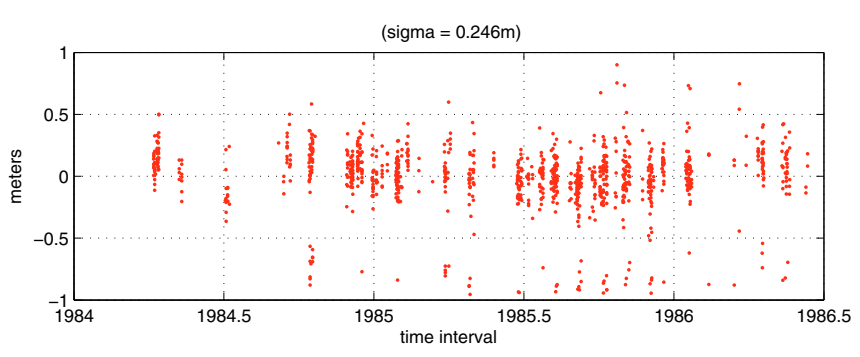

$($ sigma $=0.051 \mathrm{~m})$

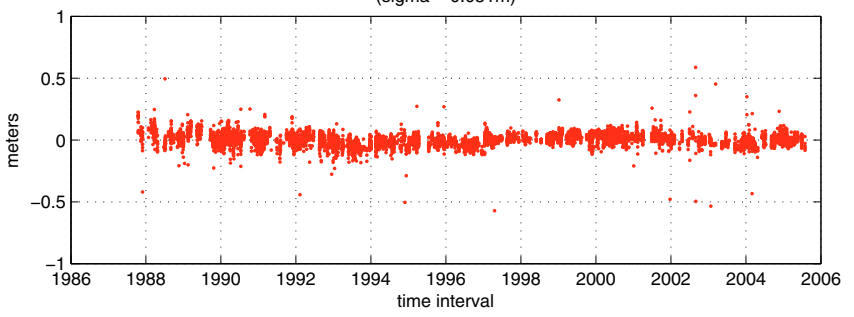

Fig. 2. LLR residuals in meters at the Grasse station (1984-2005).

We separated the residuals into two parts for each station, in order to show the improvement of the accuracy over the period of observation (Fig. 2: Grasse station - upper: LLR residuals 1984-1986 - lower: LLR residuals 1987-2005 - Fig. 3: McDonald station - upper: LLR residuals 1969-1986 - lower: LLR residuals 1987-2006). Table 1, Fig. 2 and Fig 3 show the improvement of the accuracy since 1987 , which is due to the improvements in the instruments.

\section{Lunar laser ranging data analysis: fitted parameters}

To represent the planetary and lunar motions, two different approaches are possible: a numerical approach (numerical integration), such as the JPL solution, and an analytical approach.

In our work, we have used the ELP2000 solution and adopted the strategy composed of the two following steps:

(i) once the residuals have been obtained as explained in the previous section, we determined the following parameters:

- the orbital parameters of the Moon and of the Earth-Moon barycenter in the BCRS, which include the mean motions of the Moon and of the Earth-Moon barycenter as well as the tidal secular acceleration of the Moon;

- the orientation angles of the lunar orbit at J2000.0 with respect to the GCRS;

- the main free libration parameters of the Moon in the BCRS;

- the coordinates of the reflectors in the selenocentric reference system.

Once we determined these parameters, we fixed them and checked their stability.
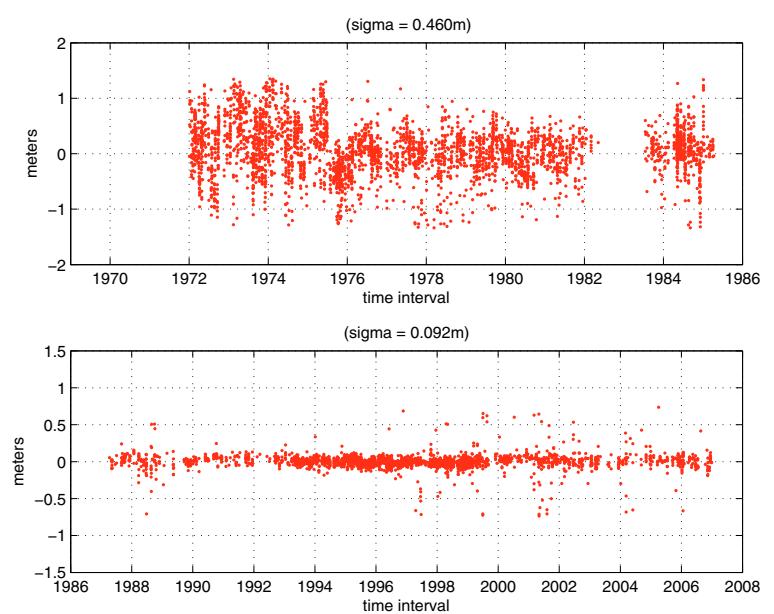

Fig. 3. LLR residuals in meters for the McDonald station (1969-2006).

The aim of this step is to determine the most stable lunar orbit over the whole interval of LLR observations in order to provide the best possible dynamical reference frame available at each date of the LLR observations. That orbit has been determined using the very complete lunar ephemeris ELP2000 and fitting the orbital parameters described above to the whole set of available LLR observations. The dynamical reference frame thus realized over the interval of LLR observations benefits from the quality of both the ELP2000 theory and the longest interval of LLR observations;

(ii) Then, we calculated the corrections to the celestial pole coordinates as described in the next section.

The aim of this final step was to determine a time series of GCRS celestial pole coordinates $D X(t)$ and $D Y(t)$ having the best possible resolution with sufficient precision. The reference to the GCRS was obtained through the link to the GCRS of the dynamical reference frame as determined in the first step.

\subsection{Calculation of the celestial pole offsets with respect to the IAU2006-2000A precession-nutation}

LLR residuals have been used to fit the corrections to the $X$ and $Y$ coordinates of the CIP in the GCRS with respect to the IAU2006-2000A model of precession nutation (cf. Mathews et al. 2002; Capitaine et al. 2003). For this analysis, the partial derivatives of the matrix transformation for the GCRS motion of the CIP are calculated (for more details see Capitaine \& Gontier 1991, and IERS Conventions 2003).

\subsubsection{Partial derivatives of the observables with respect to the $X, Y$ variables}

To express the components of $\boldsymbol{E S}$ in the GCRS, we have to apply the following transformation:

$\boldsymbol{E} \boldsymbol{S}_{\mathrm{GCRS}}=\mathbf{M} \cdot \boldsymbol{E} \boldsymbol{S}_{\mathrm{ITRS}}$

where $\mathbf{M}$ is the matrix transformation from the ITRS to the GCRS. It can be expressed as the product of three matrix transformations.

$\mathbf{M}=\mathbf{Q}(t) \cdot \mathbf{R}(t) \cdot \mathbf{W}(t)$

W being the matrix transformation for the motion of the CIP in the ITRS, $\mathbf{R}$ the matrix transformation for the Earth rotation 
and $\mathbf{Q}$ the matrix transformation for the motion of the CIP in the GCRS.

$\mathbf{Q}$ can be written as:

$\mathbf{Q}=\mathbf{P N}(X, Y) \cdot \mathbf{R}_{3}(s)$,

PN being the precession nutation matrix and $\mathbf{R}_{3}(s)$ the rotation matrix around the $z$-axis with the angle $s$, which is the CIO locator.

Hence, the partial derivatives with respect to the $X$ coordinate are:

$\frac{\delta \mathbf{Q}}{\delta X}=\frac{\delta \mathbf{P N}(X, Y)}{\delta X} \cdot \mathbf{R}_{3}(s)$, where

$\frac{\delta \mathbf{P N}(X, Y)}{\delta X}=\left(\begin{array}{ccc}-2 a X-\frac{X^{3}}{4} & -a Y-\frac{X^{2} Y}{4} & 1 \\ -a Y-\frac{X^{2} Y}{4} & -\frac{X Y^{2}}{4} & 0 \\ -1 & 0 & -2 a X-\frac{X\left(X^{2}+Y^{2}\right)}{4}\end{array}\right)$,

with: $a=1 / 2+1 / 8\left(X^{2}+Y^{2}\right)$ with an accuracy of $1 \mu$ as.

The partial derivatives with respect to the $Y$ coordinate are:

$\frac{\delta \mathbf{Q}}{\delta Y}=\frac{\delta \mathbf{P N}(X, Y)}{\delta Y} \cdot \mathbf{R}_{3}(s)$, where

$\frac{\delta \mathbf{P N}(X, Y)}{\delta Y}=\left(\begin{array}{ccc}-\frac{X^{2} Y}{4} & -a X-\frac{X Y^{2}}{4} & 0 \\ -a X-\frac{X Y^{2}}{4} & -2 a Y-\frac{Y^{3}}{4} & 1 \\ 0 & -1 & -2 a Y-\frac{Y\left(X^{2}+Y^{2}\right)}{4}\end{array}\right)$.

Finally, the partials of $\mathbf{M}$ with respect to $X$ and $Y$ are:

$\frac{\partial \mathbf{M}}{\partial X}=\frac{\partial \mathbf{Q}}{\partial X} \cdot \mathbf{R}(t) \cdot \mathbf{W}(t)$

$\frac{\partial \mathbf{M}}{\partial Y}=\frac{\partial \mathbf{Q}}{\partial Y} \cdot \mathbf{R}(t) \cdot \mathbf{W}(t)$.

The partial derivatives of the calculated time delay with respect to $X$ and $Y$ can be obtained from:

$$
\begin{aligned}
\frac{\partial \Delta t_{\mathrm{c}}}{\partial X} & =\frac{2}{c}\left[\frac{\boldsymbol{D}}{D} \cdot\left(-\frac{\partial \mathbf{M}}{\partial X}\right) \boldsymbol{E} \boldsymbol{S}_{\mathrm{ITRS}}\right] \\
\frac{\partial \Delta t_{\mathrm{c}}}{\partial Y} & =\frac{2}{c}\left[\frac{\boldsymbol{D}}{D} \cdot\left(-\frac{\partial \mathbf{M}}{\partial Y}\right) \boldsymbol{E} \boldsymbol{S}_{\mathrm{ITRS}}\right] .
\end{aligned}
$$

\subsubsection{Choice of an appropriate windowing for the calculation of the corrections to the $D X, D Y$ coordinates}

We have looked for the most appropriate interval to estimate the corrections to the $X, Y$ coordinates of the CIP with respect to the IAU2006-2000A model, using LLR data. Many investigations have been done in order to find the most appropriate resolution and the best estimation of the parameters. We tested different time intervals. With a time interval shorter than 70 days it was not possible to make an estimation of the parameters because of the imperfect distribution of the observations. A time interval longer than 70 days did not allow a sufficient resolution. Consequently, we have applied the partial derivatives of the LLR observables (cf. Eq. (6)) for the calculation of the corrections $D X, D Y$ every 70 days and obtained a series of 175 corrections.

Figures 4 and 5 show the corrections $D X, D Y$ to the celestial pole coordinates and the corrections with their formal errors, respectively. It is clear that our estimation has a better precision since 1987, when the LLR residuals were improved. Figure 6 represents a zoom of Fig. 4 over the period 1987-2006.
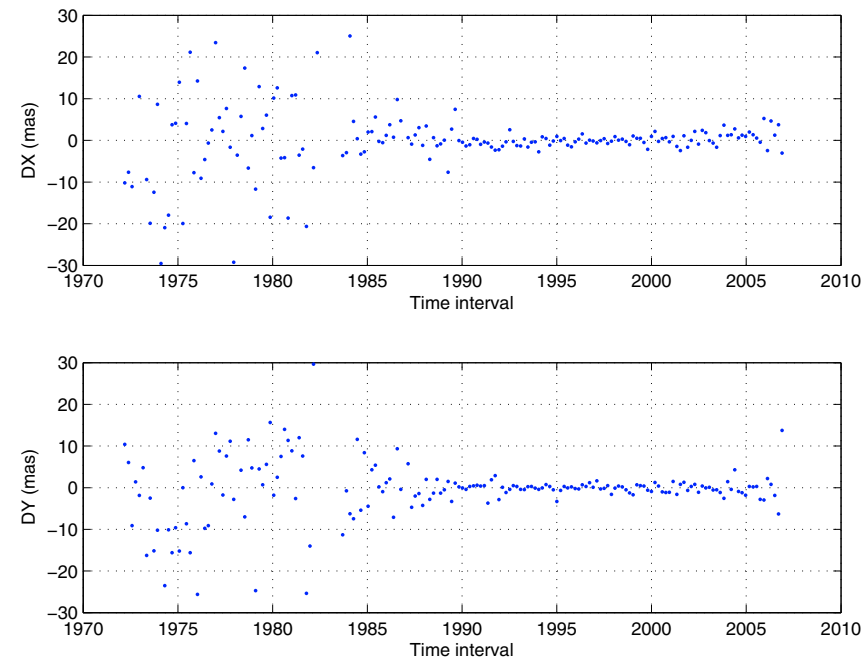

Fig. 4. Corrections to the celestial pole coordinates $(D X, D Y)$ using LLR observations (70-day resolution).
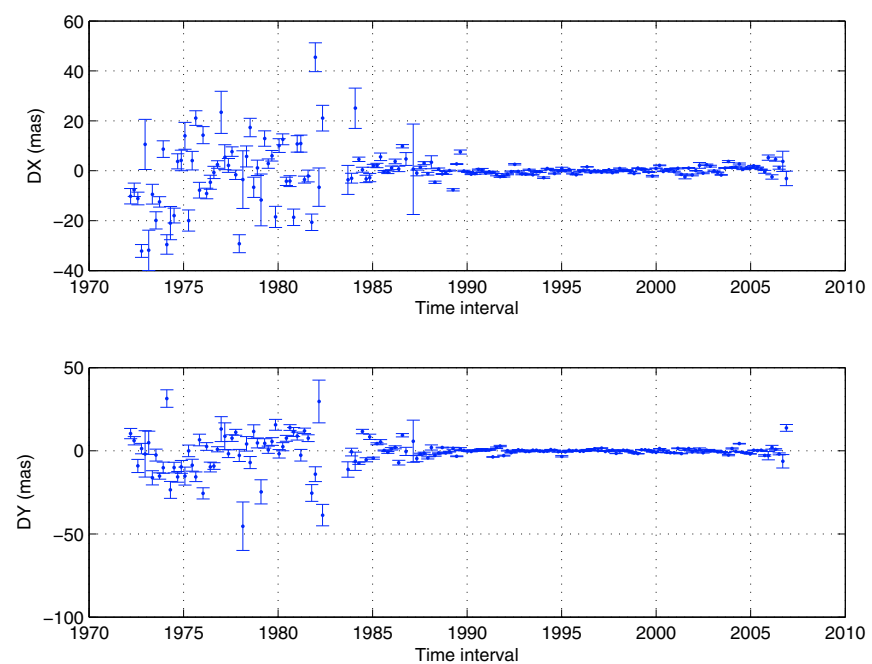

Fig. 5. Corrections to the celestial pole coordinates $(D X, D Y)$ with their formal errors using LLR observations (70-day resolution).
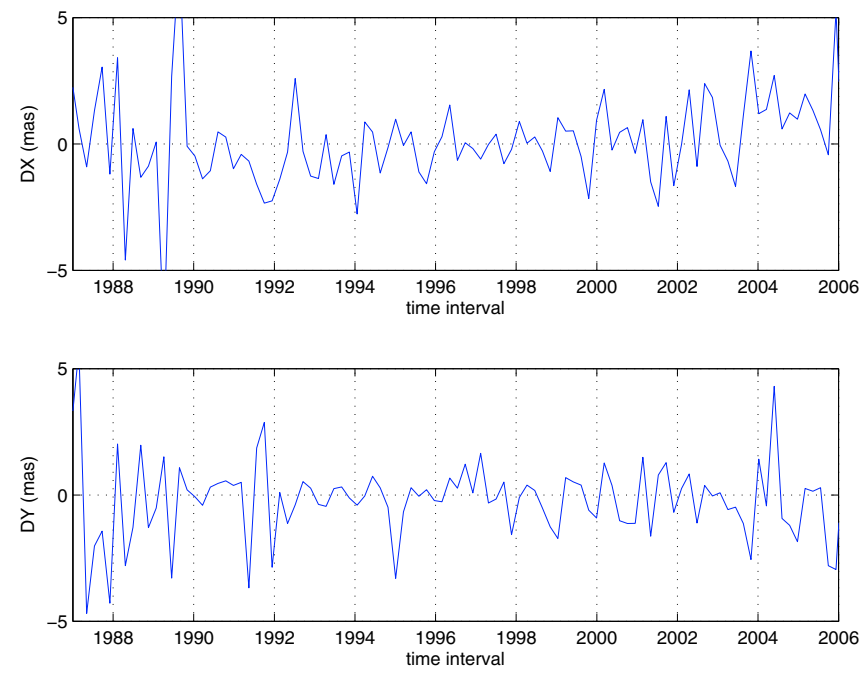

Fig. 6. Corrections to the celestial pole coordinates $(D X, D Y)$ using LLR observations over the period 1987-2006 (70-day resolution). 

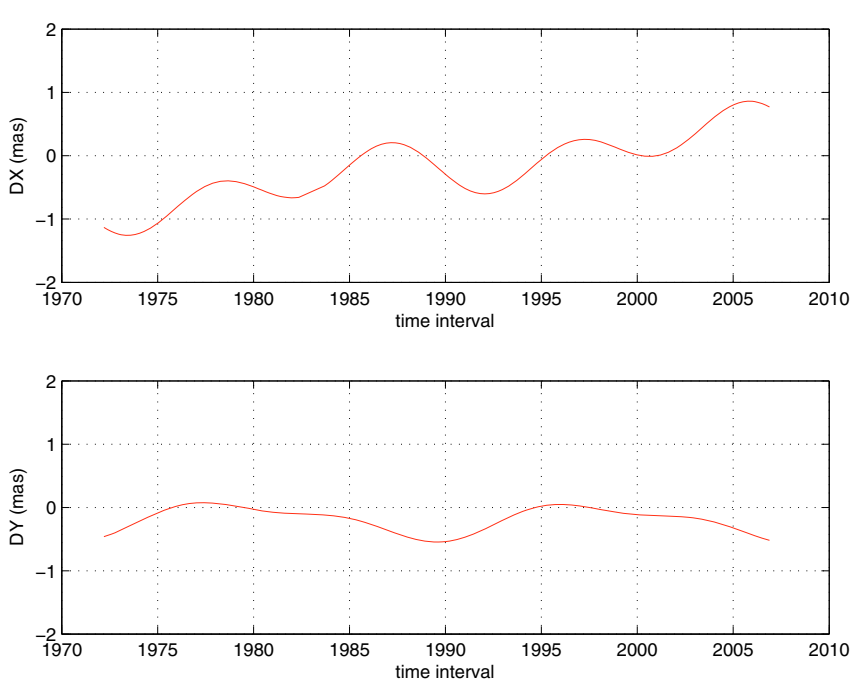

Fig. 7. Time series of the long term corrections (constant term, secular term, 18.6-yr and 9.3-yr nutation terms) for $X$ and $Y$ coordinates fitted to LLR observations.

Table 2. Numerical results for the weighted fits of periodic nutation terms (i.e 18.6-yr and 9.3-yr) plus secular and constant term for the LLR series.

\begin{tabular}{crr}
\hline \hline Terms & $D X($ mas $)$ & $D Y$ (mas) \\
\hline $\sin (18.6-\mathrm{yr})$ & $0.171 \pm 0.273$ & $0.075 \pm 0.255$ \\
$\cos (18.6-\mathrm{yr})$ & $0.121 \pm 0.144$ & $-0.241 \pm 0.143$ \\
$\sin (9.3-\mathrm{yr})$ & $0.121 \pm 0.163$ & $0.104 \pm 0.154$ \\
$\cos (9.3-\mathrm{yr})$ & $0.315 \pm 0.139$ & $-0.009 \pm 0.131$ \\
secular term (mas/cy) & $3.516 \pm 3.841$ & $-0.155 \pm 3.662$ \\
constant term & $0.163 \pm 0.153$ & $-0.220 \pm 0.145$ \\
\hline
\end{tabular}

These figures show that LLR observations allow us a determination of time series of corrections to the celestial pole coordinates (i.e. celestial pole offsets), though not with the same accuracy and resolution as with VLBI. However, the celestial pole offsets from LLR data are provided using a different approach from the ones obtained by VLBI.

\subsection{Analysis of the DX, DY time series}

In order to characterize the signal, we estimated corrections to periodic nutation terms at different periods as well as secular and constant terms using a weighted least square fit. In our calculation we first removed from the LLR series the free fore nutation (FCN) using the empirical model of Lambert (cf. IERS Conventions 2003) ${ }^{3}$.

\subsubsection{Estimation of a slope and corrections to nutation terms}

We have considered three different estimations. In all cases, we note that the uncertainties are large compared to the estimated values (see the discussion in Sect. 6); however, the uncertainties are approximately of the order of $100-300 \mu$ as, which is an improvement compared to previous studies:

(i) first, we estimated the long term components (i.e constant term, secular term, 18.6-yr and 9.3-yr nutation terms).

The numerical results for the amplitudes of the terms are shown in Table 2. Figure 7 represents the time series of

3 http://hpiers.obspm.fr/eop-pc/models/fcn/index.html
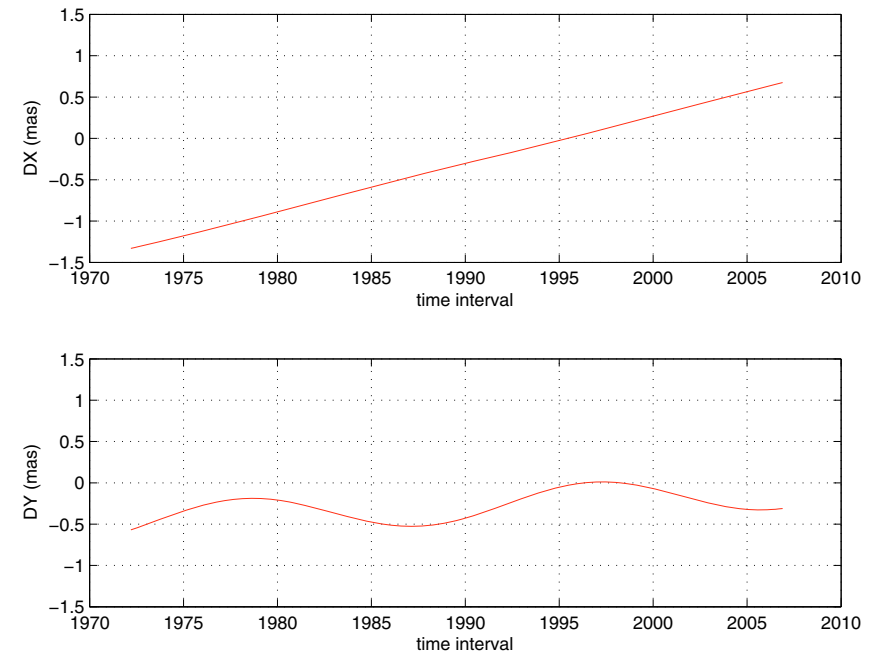

Fig. 8. Time series of the long term corrections (constant term, secular term, 18.6-yr nutation term) for $X$ and $Y$ coordinates fitted to LLR observations.

Table 3. Numerical results for the weighted fits of the 18.6-yr nutation term plus the secular and constant terms for the LLR series.

\begin{tabular}{crr}
\hline \hline Terms & $D X($ mas $)$ & $D Y$ (mas) \\
\hline sin (18.6-yr) & $0.002 \pm 0.221$ & $-0.022 \pm 0.206$ \\
cos (18.6-yr) & $0.009 \pm 0.129$ & $-0.215 \pm 0.123$ \\
secular term (mas/cy) & $5.769 \pm 3.246$ & $1.068 \pm 3.108$ \\
constant term & $0.270 \pm 0.134$ & $-0.174 \pm 0.125$ \\
\hline
\end{tabular}

Table 4. Numerical results for the weighted fits of annual and semiannual components plus the secular and constant terms for the LLR series.

\begin{tabular}{crr}
\hline \hline Terms & $D X$ (mas) & $D Y$ (mas) \\
\hline sin (annual) & $0.380 \pm 0.135$ & $0.204 \pm 0.128$ \\
cos (annual) & $-0.189 \pm 0.125$ & $-0.232 \pm 0.122$ \\
sin (semi-annual) & $0.104 \pm 0.131$ & $0.272 \pm 0.126$ \\
cos (semi-annual) & $0.128 \pm 0.120$ & $-0.168 \pm 0.113$ \\
secular term (mas/cy) & $5.597 \pm 2.093$ & $-0.108 \pm 1.989$ \\
constant term & $0.154 \pm 0.104$ & $-0.183 \pm 0.098$ \\
\hline
\end{tabular}

the long term components corresponding to the fitted coefficients of Table 2 .

Our estimates show that the correction for the 9.3-yr cosine term is larger by about a factor of 2 than for the other terms in the $X$ coordinate. The correction to the 18.6-yr cosine term is the largest one for the $Y$ coordinate;

(ii) second, we estimated the constant term, secular term and the 18.6-yr nutation term. The numerical results are shown in Table 3. It shows that the corrections for the terms are smaller than $200 \mu$ as except for the 18.6-yr cosine term in the $Y$ coordinate.

Figure 8 represents the time series of the long term components corresponding to the fitted coefficients of Table 3.

The difference between the corrections in Tables 2 and 3 are probably due to the correlation between the 18.6-yr and 9.3-yr terms as shown in Table 5 in the next paragraph;

(iii) third, we have estimated the constant term, secular term, annual and semi-annual components. The numerical results are shown in Table 4. Figure 9 represents the time series of the components corresponding to the fitted coefficients of Table 4. 

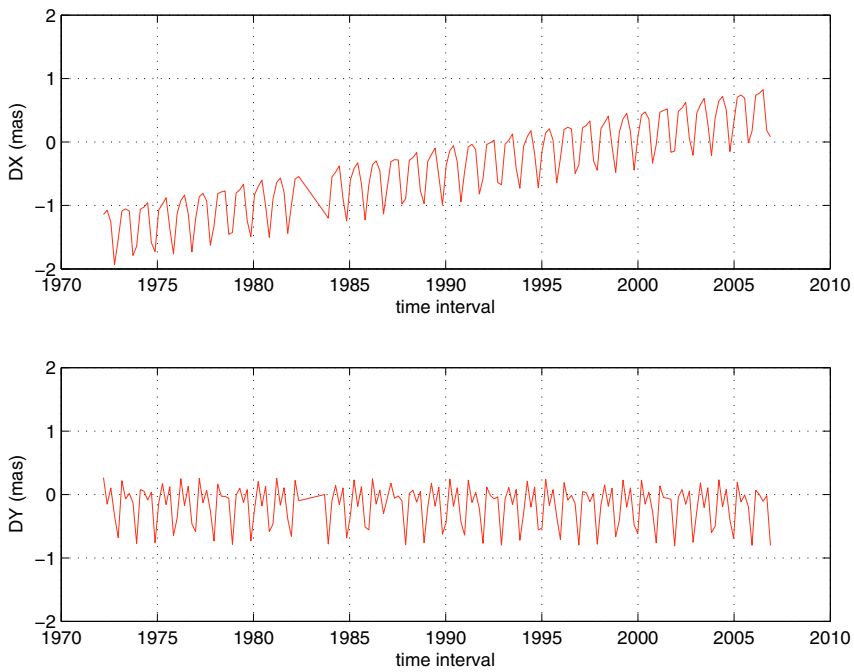

Fig. 9. Time series of the constant term, secular term, annual and semi annual terms for $X$ and $Y$ coordinates fitted to LLR observations.

Table 5. Coefficients of correlation for $D X, D Y$ coordinates corresponding to the fit of Table 2 for LLR series.

\begin{tabular}{cccc}
\hline \hline Terms & Sin (9.3-yr) & Secular term & Constant term \\
\hline $\sin (18.6-\mathrm{yr})$ & 0.6 & -0.8 & -0.7 \\
$\sin (9.3-\mathrm{yr})$ & 1 & -0.5 & \\
secular term & & 1 & 0.7 \\
\hline
\end{tabular}

In this case, the numerical results show that the largest correction is for the sine term of the annual nutation for the $X$ component, but concerning the other terms the corrections are approximately of the same order as their uncertainties.

\subsubsection{Correlation coefficients}

The coefficients of correlation corresponding to the fit of Table 2 are provided in Table 5 for the $D X, D Y$ coordinates. Only the coefficients greater than 0.5 are shown.

We can see that there is a strong correlation $(0.8)$ between the 18.6-yr sine term and the secular term and a correlation of 0.7 between the secular term and the constant term as well as between the 18.6-yr sine term and the constant term. This shows that the LLR data do not yet allow us to separate the secular term and the 18.6-yr sine nutation term as well as the constant term and the 18.6-yr sine nutation term.

Even if the uncertainties are greater than $100 \mu \mathrm{as}$, these determinations provide information about the time series obtained and their long term nutation components as well as their annual and semi annual terms.

\section{Analysis of the celestial pole offsets derived from VLBI observations}

In order to compare LLR estimations with VLBI ones, we carried out the same investigation using the IVS combined solution (ivse08q1.eops) that includes 2846 values from 1984 to 2008. The celestial pole offsets $D X, D Y$ of this solution are currently given with respect to the IAU 2000 model. Then, to be consistent with our work, we estimated the $D X, D Y$ corrections with respect to the IAU 2006-2000A model. For this determination, we used the equation (cf. Capitaine \& Wallace 2006, Eq. (41),
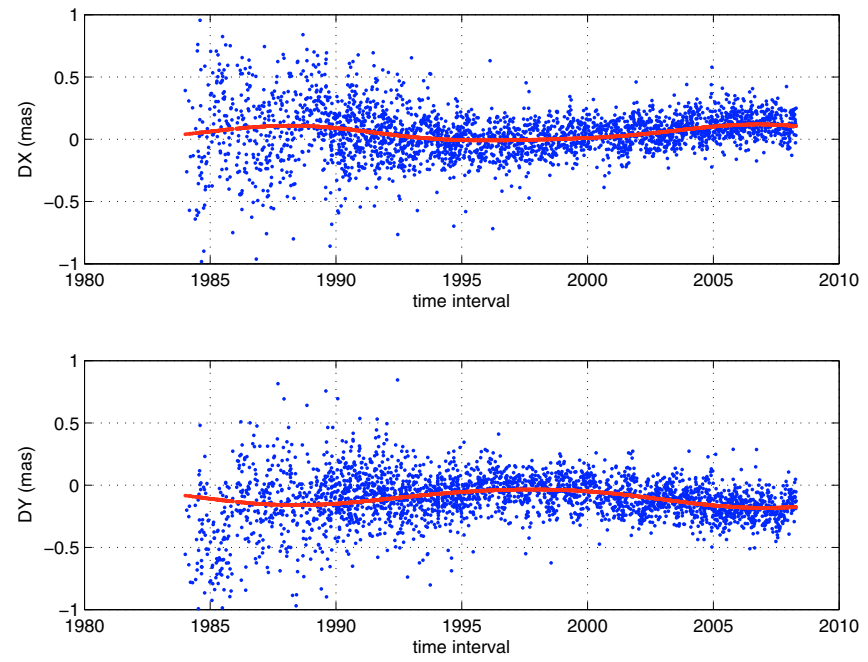

Fig. 10. $D X, D Y$ corrections after removing the FCN from VLBI (ivse08q1.eops) series w.r.t. the IAU2006-2000A model of precession nutation - the curve: time series of the long term corrections (constant term, secular term, 18.6-yr and 9.3-yr nutation terms) for $X$ and $Y$ coordinates fitted to VLBI observations.

Table 6. Numerical amplitudes of the fitted terms (i.e constant term, secular term, 18.6-yr and 9.3-yr nutation terms) for the VLBI series.

\begin{tabular}{crr}
\hline \hline Terms & $D X($ mas $)$ & $D Y$ (mas) \\
\hline sin (18.6-yr) & $0.000 \pm 0.004$ & $0.013 \pm 0.004$ \\
$\cos (18.6-y r)$ & $0.060 \pm 0.004$ & $-0.067 \pm 0.004$ \\
$\sin (9.3-y r)$ & $-0.004 \pm 0.003$ & $-0.000 \pm 0.003$ \\
$\cos (9.3-y r)$ & $0.011 \pm 0.003$ & $-0.005 \pm 0.003$ \\
secular term (mas/cy) & $0.054 \pm 0.067$ & $-0.118 \pm 0.067$ \\
constant term & $0.044 \pm 0.002$ & $-0.101 \pm 0.002$ \\
\hline
\end{tabular}

after correcting a typographical error) for the difference between the IAU2006 and IAU2000 expressions of the precession in $X$ and $Y$ (in $\mu$ as):

$$
\begin{aligned}
& X_{\mathrm{IAU} 2006}-X_{\mathrm{IAU} 2000}=155 t-2564 t^{2}+2 t^{3}+54 t^{4} \\
& Y_{\mathrm{IAU} 2006}-Y_{\mathrm{IAU} 2000}=-514 t-24 t^{2}+58 t^{3}-1 t^{4}-1 t^{5} .
\end{aligned}
$$

\subsection{Analysis of the DX, DY VLBI time series}

Once we have obtained the time series of $D X, D Y$ corrections with respect to the IAU 2006-2000A model, we perform the same analysis as in Sect. 4 after removing the FCN:

(i) first, fitting the long term components (i.e constant term, secular term, 18.6-yr and 9.3-yr nutation terms). The numerical results are printed in Table 6 . This table shows that the correction for the 18.6-yr cosine term is the largest one for the $X$ and $Y$ components. Also, our calculations show that the estimation of the amplitude of the fitted terms is determined with a precision that is better by a factor of 50 than in the LLR analysis;

Figure 10 represents the $D X, D Y$ corrections after removing the FCN from VLBI (ivse08q1.eops) series w.r.t. the IAU2006-2000A model of precession nutation and the time series of the long term components (constant term, secular term, 18.6-yr and 9.3-yr nutation terms) for $X$ and $Y$ coordinates fitted to VLBI observations;

(ii) second, we have estimated the constant term, secular term and the 18.6-yr nutation term. The numerical results are 

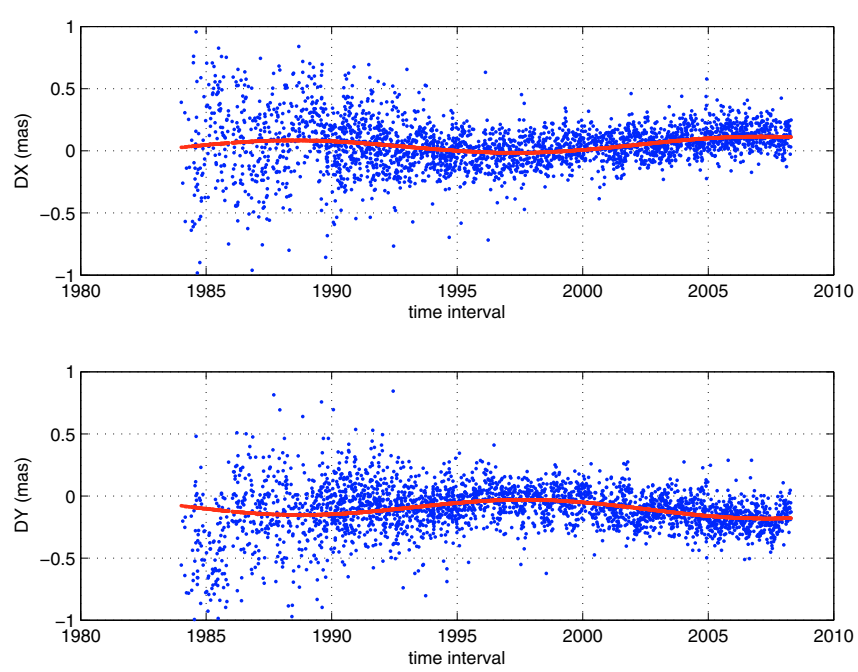

Fig. 11. $D X, D Y$ corrections after removing the FCN from VLBI (ivse08q1.eops) series w.r.t. the IAU2006-2000A model of precession nutation - the curve: time series of the long term components (constant term, secular term, 18.6-yr nutation term) for $X$ and $Y$ coordinates fitted to VLBI observations.

Table 7. Numerical amplitudes of the fitted terms (i.e constant term, secular term, 18.6-yr nutation term) for the VLBI series.

\begin{tabular}{crr}
\hline \hline Terms & $D X(\mathrm{mas})$ & $D Y(\mathrm{mas})$ \\
\hline $\sin (18.6-\mathrm{yr})$ & $-0.007 \pm 0.003$ & $0.016 \pm 0.003$ \\
$\cos (18.6-\mathrm{yr})$ & $0.057 \pm 0.004$ & $-0.066 \pm 0.004$ \\
secular term (mas/cy) & $0.164 \pm 0.060$ & $-0.155 \pm 0.060$ \\
constant term & $0.045 \pm 0.002$ & $-0.102 \pm 0.002$ \\
\hline
\end{tabular}

shown in Table 7. Figure 11 represents the time series of the long term components corresponding to the fitted coefficients of Table 7 .

The results show that there is no significant difference between case 1 and 2 (cf. Tables 6 and 7), in contrast to LLR where they are different (cf. Tables 2 and 3 ).

\subsection{Correlation coefficients}

The coefficients of correlation corresponding to the fit of Table 6 are provided in Table 8 for the $D X$ and $D Y$ coordinates. Only the correlations greater than 0.5 are given.

We can see that contrary to the LLR correlations, there is a strong correlation (approx. 0.70) between the 18.6-yr cosine term and the secular term.

\section{Discussion}

The large uncertainties of the estimated values from LLR are due to the imperfect distribution of the observations. LLR solution values for the corrections to the 18.6-yr and 9.3-yr nutation coefficients for case 1 (Table 2) are almost of the same order except for 18.6-yr sine term and 9.3-yr cosine term in the $Y$ component, where the values are smaller. However, if we compare those values with those obtained in case 2 (Table 3), they are smaller by a factor 100 than in the first case, especially for the $X$ component. This is probably due to the data span of LLR that is not yet long enough to reliably separate the 18.6-yr and 9.3-yr coefficients. Even if a $37-y r$ interval should be sufficient to allow the separation between the long periodic nutation terms from the
Table 8. Coefficients of correlation corresponding to the fit of Table 6 for VLBI series.

\begin{tabular}{cc}
\hline \hline Terms & Secular term \\
\hline $\sin (18.6 \mathrm{yr})$ & -0.5 \\
$\cos (18.6 \mathrm{yr})$ & -0.7 \\
\hline
\end{tabular}

constant and the secular term, this separation is still imperfect due to the insufficient precision of the LLR data over the interval 1969-1986.

We have also determined corrections to the annual and semiannual terms, where the values are of the same order for the $X$ and $Y$ coordinates.

LLR data have the property of being sensitive to both the ecliptic and the equator. However, comparison between the results of Sects. 4 and 5 shows that LLR data are not as robust as VLBI for the determination of the celestial pole offsets because there are fewer LLR observation stations. We do not expect agreement with VLBI results at the level of VLBI accuracy. However, we have shown that it is possible to determine celestial pole offsets from LLR observations and have specified the characteristics of this time series.

\section{Joint analysis of LLR and VLBI data}

In this section, we present a joint analysis of LLR and VLBI data combining the two series of celestial pole offsets $D X, D Y$. The principal reason for doing this combination is the complementarity of these two series; the combined series benefits from the accuracy of VLBI, as well as the advantage of the LLR by its longer time series and its different approach for determining the celestial pole offsets.

In a first step, the celestial pole offsets of each space-geodetic technique were analyzed separately. Statistical analysis were applied in order to determine an optimal weight for both series. For the VLBI series, we applied the Allan variance after removing all the known systematic errors (i.e the FCN, the 18.6-yr nutation term and 9.3-yr nutation term). LLR series were compared to the $\mathrm{C} 04$ series of the IERS which were considered as the reference series. Many tests have been performed in particular to check that LLR series are in the reference system of the $\mathrm{C} 04$ series and to rescale our standard deviation. We obtained a bias of $121 \mu$ as in $X$ and $-181 \mu$ as in $Y$ with a standard deviation of about 1 mas. This means that the series is actually in the C04 reference system. Concerning the standard deviation, the statistical tests demonstrated that our formal errors are not realistic, and have to be multiplied by a factor of 2 .

In order to combine both series, we computed a weighted mean VLBI series at 70 days interval (i.e. the same time interval as in the LLR data). The series obtained has 127 values; it is represented in Fig. 12.

The principle of the combination used is the following: we have stacked the two series, the VLBI one being more accurate and the LLR one being longer, and choosing the corresponding optimal weights. Several tests have been made in order to determine the optimal values for both series. Finally, we adopted the strategy described below:

- for the non common periods (1969-1985 and 2006-2008), we retained the weights associated to the corrections $D X, D Y$ of each series.

- for the common period (1985-2006), we calculated the relative weight taking into account the two techniques, using the 

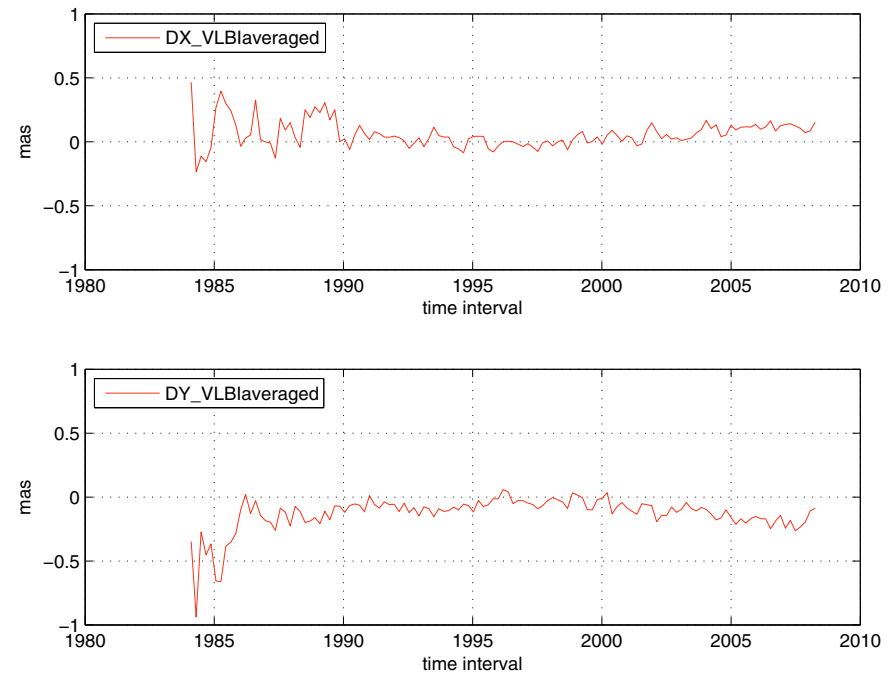

Fig. 12. $D X$ and $D Y$ corrections after removing the FCN of the VLBI time series w.r.t. the IAU 2006-2000A model of precession nutation averaged over 70 days.

following:

$$
\begin{aligned}
& \text { Weight }_{\mathrm{LLR}}=\left(\frac{1}{\sigma_{\mathrm{LLR}}^{2}}\right) /\left(\frac{1}{\sigma_{\mathrm{LLR}}^{2}}+\frac{1}{\sigma_{\mathrm{VLBI}}^{2}}\right) \\
& \text { Weight }_{\mathrm{VLBI}}=\left(\frac{1}{\sigma_{\mathrm{VLBI}}^{2}}\right) /\left(\frac{1}{\sigma_{\mathrm{LLR}}^{2}}+\frac{1}{\sigma_{\mathrm{VLBI}}^{2}}\right) .
\end{aligned}
$$

The combined solution was obtained using the following formula:

$$
\begin{aligned}
& D X_{\text {combined }}=\text { Weight }_{\mathrm{LLR}} \times D X_{\mathrm{LLR}}+\text { Weight }_{\mathrm{VLBI}} \times D X_{\mathrm{VLBI}} \\
& D Y_{\text {combined }}=\text { Weight }_{\mathrm{LLR}} \times D Y_{\mathrm{LLR}}+\text { Weight }_{\mathrm{VLBI}} \times D Y_{\mathrm{VLBI}}
\end{aligned}
$$

The results obtained from the combination are represented in Fig. 13. The present large curves at the beginning (i.e. 1972-1985) are the celestial pole offsets derived from the LLR observations over the period (1972-1985). We have seen that over this period of time the LLR observations are less precise (see Fig. 2, Fig. 3), which is why our determination is more noisy over this period. But the advantage is to have a longer time series because there are no VLBI observations over this period of time (before 1985).

If we zoom in on the common period and show only the weighted mean VLBI series and the combined series (Fig. 14), we can see that there are cases where some signal appears in the combined solution, while it does not appear in the corresponding VLBI series. See for example $D Y$ over the period 1998-2001 and $D X$ over the period 1996-1998. This may indicate that the combined series reveals details that do not appear in the VLBI series alone.

\section{Concluding remarks}

In this paper, we have demonstrated the possibility of determining celestial pole coordinates using LLR observations. This determination is not as accurate nor as dense as with VLBI observations, but its advantage is its different approach for determining the celestial reference system, thus, the celestial pole coordinates. So far, only the VLBI technique has provided this kind of determination. This work provides some external constraints
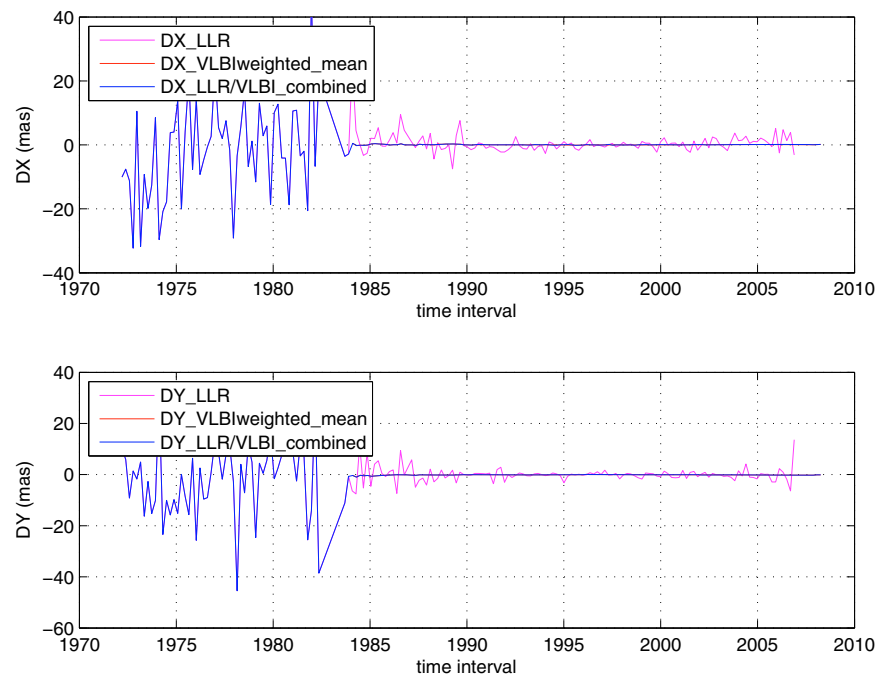

Fig. 13. $D X, D Y$ corrections after removing the FCN from LLR/VLBI w.r.t. the IAU2006-2000A model of precession nutation over the period 1969-2008.
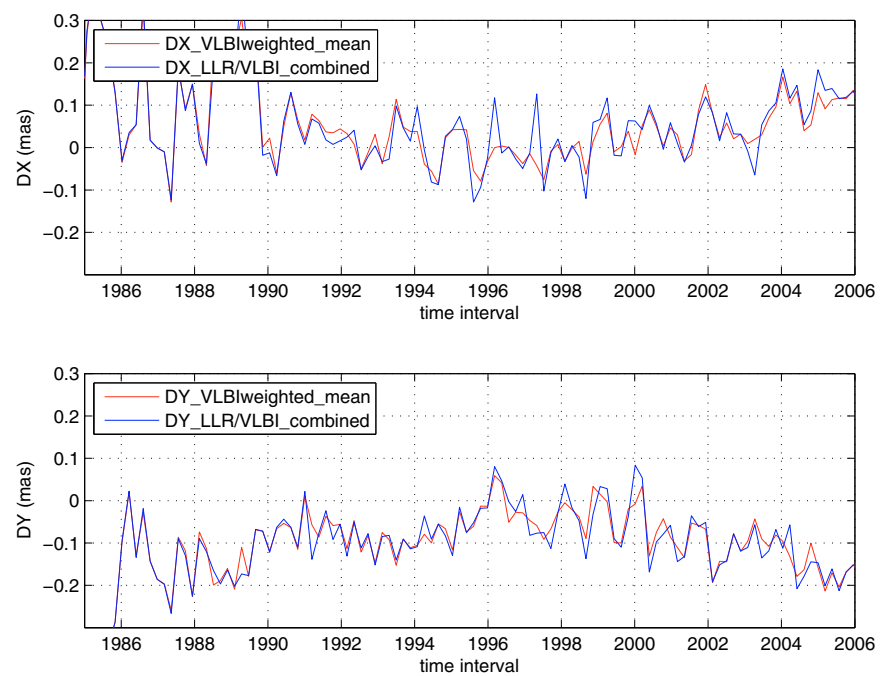

Fig. 14. $D X, D Y$ corrections after removing the FCN from LLR/VLBI w.r.t. the IAU2006-2000A model of precession nutation over the period 1984-2008.

to the celestial pole coordinates derived from VLBI observations. Moreover, the determination of the long-period nutation terms shows an improvement with respect to previous studies.

The comparison of the time series $D X, D Y$ derived from LLR observations with the C04 series has demonstrated the consistency of our calculation.

The combination of LLR and VLBI series can be stronger than either individual time series. The whole series completes the VLBI series and allows us to have a time series of celestial pole offsets over a longer interval. Further studies are necessary in order to allow a physical interpretation of the results. In the future, more LLR observations with a better resolution will offer possibilities to improve the LLR/VLBI combined series and thus the determination of the 18.6-yr nutation term and consequently of the Earth's dynamical parameters.

Acknowledgements. This work has been done in the framework of the Groupe de Recherches de Géodésie Spatiale (GRGS). We are grateful to J. Chapront and G. Francou (SYRTE, Observatoire de Paris, GRGS) for their advice and invaluable help in using and analyzing LLR data, and E. Samain (GRGS/OCA) 
for providing useful information on the LLR data. We are also grateful to J.M. Lemoine (GRGS/CNES), Ch. Bizouard, S. Bouquillon, D. Gambis, S. Lambert and J.Y. Richard (SYRTE, Observatoire de Paris, GRGS) for helpful discussion. We also acknowledge the contribution of the ILRS and IVS communities for measurements and availability of the data, and also for their continuing efforts. We thank the anonymous referee for valuable suggestions.

\section{References}

Capitaine, N., \& Gontier, A. M. 1991, in Reference Systems, ed. J. A. Hughes, C. A. Smith, \& G. H. Kaplan, Proc. IAU Coll., 127, 77
Capitaine, N., \& Wallace, P. T. 2006, A\&A, 450, 855

Capitaine, N., Wallace, P. T., \& Chapront, J. 2003, A\&A, 412, 567

Chapront-Touzé, M., \& Chapront, J. 1983, A\&A, 124, 50

Chapront, J., Chapront-Touzé, M., \& Francou, G. 1999, A\&A, 343, 624

Chapront, J., Chapront-Touzé, M., \& Francou, G. 2002, A\&A, 387, 700

Charlot, P., Sovers, O. J., Williams, J. G., et al. 1995, AJ, 109, 1, 418

Folkner, W. M., Charlot, P., Finger, M. H., et al. 1994, A\&A, 287, 279

Mathews, P. M., Herring, T. A., \& Buffet, B. A. 2002, J. Geophys. Res., 107(B4), 10.1029/2001JB00390

Moons, M. 1982, Moon and the Planets, 27, 257

Samain, E., Mangin, J. F., Veillet, C., et al. 1998, A\&A, 130, 235

Williams, J. G., Newhall, X. X., \& Dickey, J. O. 1991, A\&A, 241, L9 